\title{
Left Ventricular Morphology in Patients with Aortic Coarctation and Bicuspid Aortic Valve: Novel Insights from a Statistical Shape Modelling Framework
}

\section{Froso Sophocleous}

University of Bristol

\section{Lucy Standen}

University of Bristol

Gemina Doolub

University Hospitals Bristol and Weston NHS Foundation Trust

\section{Reem Laymouna}

University Hospitals Bristol and Weston NHS Foundation Trust

Chiara Bucciarelli-Ducci

University of Bristol

\section{Massimo Caputo}

University of Bristol

\section{Nathan Manghat}

University Hospitals Bristol and Weston NHS Foundation Trust

\section{Mark Hamilton}

University Hospitals Bristol and Weston NHS Foundation Trust

\section{Stephanie Curtis}

University Hospitals Bristol and Weston NHS Foundation Trust

Giovanni Biglino ( $\sim$ g.biglino@bristol.ac.uk)

University of Bristol

\section{Research Article}

Keywords: Left ventricle, congenital heart disease, statistical shape modelling, sphericity, ventricular strain, bicuspid aortic valve, aortic coarctation, computational modelling.

Posted Date: January 5th, 2022

DOI: https://doi.org/10.21203/rs.3.rs-1127760/v1 
License: (c) (i) This work is licensed under a Creative Commons Attribution 4.0 International License. Read Full License 


\section{Abstract \\ Background}

The functional implications of left ventricular (LV) morphological characterization in congenital heart disease (CHD) are not widely explored. This study qualitatively and quantitatively assessed LV shape associations with a) LV function and b) thoracic aortic morphology in patients with aortic coarctation (CoA) with/without bicuspid aortic valve (BAV).

\section{Methods}

A statistical shape modelling (SSM) framework was employed to analyse three-dimensional (3D) LV shapes from cardiac magnetic resonance (CMR) data in isolated CoA $(n=25), \operatorname{CoA}+B A V(n=30)$, isolated BAV $(n=30)$, and age-matched healthy controls $(n=25)$. Average 3D templates and deformations were computed. Correlations between shape data and CMR-derived morphometric parameters (i.e. sphericity, conicity) or global and apical strain values were assessed to elucidate possible functional implications. The relationship between LV shape features and arch architecture was also explored.

\section{Results}

The LV template was shorter and more spherical in CoA patient and LV sphericity was associated ( $p \leq 0.04$ ) with lower global longitudinal, radial and circumferential strain, irrespective of the presence of aortic stenosis and/or regurgitation. Conversely, LV strain was not associated with arch architecture.

\section{Conclusions}

Differences in LV morphology were observed between CoA and BAV patients. Increasing LV sphericity was associated with reduced strain, independent of aortic arch architecture and functional aortic valve disease.

\section{Background}

The ability of the heart to change its shape and function in order to maintain normal cardiac output has been characterized as "remodelling", which can either be adaptive or maladaptive, acute or chronic[1]. Left ventricular (LV) morphology has been proposed as an important marker of remodelling and related to clinical outcomes, with adverse remodelling being a strong predictor of cardiovascular disease[2-4]. The LV geometry plays a critical role in the mechanics of the heart, and as early as 1892 , Wood used a spherical model to quantitatively assess this relationship[5]. Three-dimensional (3D) patient-specific anatomical information can be derived by non-invasive imaging techniques, such as cardiovascular magnetic resonance (CMR) imaging. This allows further exploration of LV morphology using tools such 
as statistical shape modelling (SSM), which our group has previously applied to capture apical ballooning in Takotsubo cardiomyopathy[6]. Here we apply this methodology to congenital heart disease, particularly to aortic coarctation (CoA) and bicuspid aortic valve (BAV) disease, which have a prevalence of $3 / 10,000$ and $1-2 \%$ respectively[7, 8]. Patients with CoA can have BAV in up to $85 \%$ of cases, while both diseases can result in aortic vasculopathy, which is associated with morbidity and mortality in young patients with $\mathrm{CHD}[7-9]$.

To our knowledge, only one study has described LV morphology in CoA or BAV, assessing LV sphericity pre- and post-stent implantation in CoA patients[10]. Clinical observations suggest that CoA patients tend to have rounder LVs, but this has not been assessed quantitatively, nor have possible functional implications of this characteristic been explored. Thus, this study aims to assess LV morphology in patients with CoA and/or BAV, using CMR-derived sphericity and conicity indices, and by means of SSM[11]. Morphological quantification through SSM allows exploring possible associations between morphological features and functional implications, such as differences in CMR-derived global and apical strain indices. In addition, previous work from our group suggested potentially unfavourable aortic arch configurations in patients with BAV and repaired $\mathrm{CoA}[12]$ and so this study also aimed to explore possible associations between LV morphological features and aortic arch architecture.

\section{Methods}

\section{Patient population}

This was a retrospective single-centre study, screening patients who underwent clinical cardiovascular magnetic resonance (CMR) examination at a large tertiary centre between 2007 and 2020. Data were acquired at 1.5 T (Avanto, Siemens Healthineers, Erlangen, Germany) and patients for the morphological $3 D$ analysis were selected from databases of $n=521$ BAV patients, $n=633$ CoA patients and $n=435$ patients without structural heart disease (serving as healthy controls). Primary exclusion criteria were: connective tissue disorders (including Marfan, Turner and Ehlers Danlos syndromes), any concomitant either complex or moderate CHDs (including Shone's complex, tetralogy of Fallot, transposition of the great arteries and Ebstein's anomaly), unrepaired CoA, pseudo-CoA, Kawasaki disease, previous operation of aorta (apart from CoA repair) and/or valve replacement, valvotomy or aortic arch reconstruction, reduced ejection fraction (EF) $<40 \%$, and pregnancy. Further exclusion criteria included sub-optimal image quality or absence of cine $\mathrm{CMR}$ images of the left ventricle required for 3D shape reconstruction. We selected patients between 19 and 75 years of age and created four groups: i.e. isolated CoA, CoA+BAV, isolated BAV and healthy controls. Ethical approval was not required by the University Hospitals Bristol \& Weston. Research \& Innovation (R \& I) Department for this study. Informed consent was given by all patients for research use of images at the time and as part of the CMR scan consent. The study was carried out in accordance to local protocols and regulations.

Demographic variables gathered from clinical records included sex, age and body mass index. Anatomical variables included aortic valve morphology, classified according to the pattern of coronary 
leaflet non-separation. Functional variables included presence of functional aortic valve disease (i.e. aortic regurgitation and/or aortic stenosis, classified according to the European Association of Echocardiography[13]), presence and severity of CoA as classified in the literature[14], history of hypertension, and CMR-derived EF, end-diastolic volume (EDV), end-systolic volume (ESV), stroke volume (SV), and LV mass, collected from clinical CMR reports.

\section{Morphological analysis}

A SSM framework was applied to assess the LV shape, as a primary aim of this study (the methodological workflow is summarised in Figure 1). Technical SSM details are reported elsewhere[15]. Cine images in short-axis view were collected for all cases and segmented creating 3D volume meshes of LV endocardium at end-diastole using open-source software (Segment v3.0, Medviso, Lund, Sweden). The 3D shapes were then imported into Mimics (Mimics Research v.21.0, Materialise NV, Leuven, Belgium) in stl format, where they were consistently cut at the apex and base, uniformly remeshed using a factor of 4 $\mathrm{mm}$ (3matic Research v.13.0, Materialise), and registered on top of each other based on the barycentre to reduce possible alignment bias and speed-up the analysis. The 3D meshes were then imported into Deformetrica software (www.deformetrica.org) to compute the average shape of the population, or 'template', as well as the shape variation around it, or 'shape modes'[15]. Key analysis parameters were a) model stiffness, set at $35 \mathrm{~mm}$ ( $\lambda_{\text {diffeos }}$ parameter, bigger values result in "stiffer", i.e. less elastic, deformations that capture more global shape features) and b) model resolution, set at $11 \mathrm{~mm}\left(\lambda_{\text {surface }}\right.$ parameter, bigger values result in neglecting small shape features). The shape templates provide qualitative data, while the shape vectors provide quantitative data for dominant morphological features (or shape modes). Shape modes were extracted from principal component analysis in order to statistically correlate shape deformations with parameters of interest, i.e. sphericity and conicity indices, global and apical strains, and aortic arch shape. On top of the global analysis, all groups were separately processed, using the same parameters, allowing for visual differences to be displayed.

Sphericity and conicity measurements were averaged from CMR cine 2-and 4-chamber imaging views. Sphericity was measured as short to long axis ratio, while conicity was measured as apical to short axis ratio, with apical axis being the diameter of the best fitting sphere to the apex as described in[11], (see Figure 2A).

In order to assess possible associations between arch architecture and LV morphology, 3D whole heart or angiographic sequences were used to reconstruct 3D aortic arch models (Mimics) for all patients except

for healthy controls, as 3D sequences were not available in this group. The 3D aortic models were used to measure aortic gothicity (based on height/width ratio, H/W) and aortic tortuosity (automatically calculated using Vascular Modelling Toolkit, Orobix, Bergamo, Italy), (see Figure 2B).

\section{Deformation analysis}

Regional tissue tracking features in three directions (longitudinal, radial, and circumferential) were automatically computed on a 16-segment model and averaged to provide global peak longitudinal strain 
(GLS), global peak radial strain (GRS), global peak circumferential strain (GCS). The endocardial and epicardial contouring were carefully delineated from the cine images throughout the cardiac cycle. Apical strain indices were also measured. The feature tracking analysis was performed in CVI42 (Circle Cardiovascular Imaging, Calgary, AB, Canada).

\section{Statistical analysis}

Statistical analysis was run with R Studio (Vienna, Austria). Group differences for continuous variables were tested using ANOVA or Kruskal-Wallis test (with Dunn's test post hoc) depending on the normality of the distribution. Linear regression analysis was performed to verify the association between variables, including between SSM-derived shape modes and demographic, anatomical and functional variables. Multiple regression analysis was run to assess the effect of aortic arch architecture (i.e. tortuosity and gothicity), aortic regurgitation and aortic stenosis on the relationship between LV morphology and strain. For the purpose of the analysis and considering the available sample size, aortic regurgitation and stenosis severity were treated as categorical variables (none or mild $=0$, moderate and severe $=1$ ). Alpha was set at 0.05 .

\section{Results}

Patient demographic and clinical characteristics are reported below in Table 1 and CMR measurements are reported in Table 2. The isolated CoA group had higher prevalence of history of hypertension $(p=0.03)$. The majority of BAV patients had right-left leaflet non-separation. Also, CoA+BAV patients had increased CoA severity compared to isolated CoA (moderate or severe CoA in $37 \%$ vs $21 \%, p=0.01$ ) and, overall, very few patients presented with severe aortic stenosis or regurgitation. All groups had normal EF around $65 \%$, and patients with BAV had larger LV volumes and higher LV mass (Table 2). Interestingly, patients with CoA showed higher sphericity index $(p=0.02)$ whilst no group difference was found for arch gothicity or tortuosity ( $p=0.28$ and $p=1$, respectively).

Three-dimensional templates, calculated for the whole population and the four groups separately, are shown in Figure 3, with atlas error $\leq 0.4 \mathrm{~mm}$. As it can be qualitatively appreciated, the template for isolated CoA patients is the shortest and more spherical compared to the others, followed by BAV-CoA and then isolated BAV, compared to the more elongated healthy controls. 
Table 1

Patient demographic and clinical characteristics.

\begin{tabular}{|c|c|c|c|c|c|}
\hline & $\begin{array}{l}\text { Isolated CoA } \\
(\mathrm{N}=25)\end{array}$ & $\begin{array}{l}\text { CoA+BAV } \\
(N=30)\end{array}$ & $\begin{array}{l}\text { Isolated BAV } \\
(\mathrm{N}=30)\end{array}$ & $\begin{array}{l}\text { Controls } \\
(\mathrm{N}=25)\end{array}$ & $\begin{array}{l}\mathrm{p}- \\
\text { values }\end{array}$ \\
\hline $\operatorname{Sex}(n, \% M)$ & $12,48 \%$ & $16,53 \%$ & $16,53 \%$ & $12,48 \%$ & $p=0.96$ \\
\hline Age (years) & $37 \pm 12,(19-66)$ & $\begin{array}{l}43 \pm 8,(31- \\
57)\end{array}$ & $46 \pm 14,(20-75)$ & $\begin{array}{l}45 \pm 11,(27- \\
64)\end{array}$ & $p=0.03$ \\
\hline $\mathrm{BMI}\left(\mathrm{kg} / \mathrm{m}^{2}\right)$ & $27 \pm 6,(20-42)$ & $\begin{array}{l}27 \pm 5,(17- \\
38)\end{array}$ & $26 \pm 5,(17-39)$ & $\begin{array}{l}26 \pm 4,(21- \\
34)\end{array}$ & $p=0.97$ \\
\hline $\begin{array}{l}\text { Presence of } \\
\text { hypertension }(n, \%)\end{array}$ & $18,72 \%$ & $11,37 \%$ & $13,48 \%$ & - & $p=0.03$ \\
\hline \multirow[t]{3}{*}{ Valve Phenotype $(\mathrm{n}, \%)$} & \multirow[t]{3}{*}{-} & RL: $26,87 \%$ & $\mathrm{RL}: 19,63 \%$ & \multirow[t]{3}{*}{-} & \\
\hline & & RNC: $3,10 \%$ & RNC: $11,37 \%$ & & \\
\hline & & LNC: $1,3 \%$ & LNC: $0,0 \%$ & & \\
\hline \multirow[t]{4}{*}{ CoA severity, $(\mathrm{n}, \%)$} & $\begin{array}{l}\text { Nones: } 11 \\
46 \%\end{array}$ & $\begin{array}{l}\text { Nones: } 12, \\
40 \%\end{array}$ & \multirow[t]{4}{*}{-} & \multirow[t]{4}{*}{-} & \\
\hline & Mild: 8, 33\% & Mild: 7, 23\% & & & \\
\hline & $\begin{array}{l}\text { Moderate: } 1 \\
4 \%\end{array}$ & $\begin{array}{l}\text { Moderate: } 3 \\
10 \%\end{array}$ & & & \\
\hline & Severe: $4,17 \%$ & $\begin{array}{l}\text { Severe: } 8 \\
27 \%\end{array}$ & & & \\
\hline \multirow[t]{4}{*}{$\begin{array}{l}\text { Aortic stenosis severity, } \\
(\mathrm{n}, \%)\end{array}$} & $\begin{array}{l}\text { None: } 25 \text {, } \\
100 \%\end{array}$ & $\begin{array}{l}\text { None: } 21 \\
70 \%\end{array}$ & \multirow{4}{*}{$\begin{array}{l}\text { None: } 15,50 \% \\
\text { Mild: } 3,10 \% \\
\text { Moderate: } 8 \\
28 \% \\
\text { Severe: 4, 14\% }\end{array}$} & $\begin{array}{l}\text { None: } 25, \\
100 \%\end{array}$ & \\
\hline & Mild: 0, 0\% & Mild: 6, 21\% & & Mild: $0,0 \%$ & \\
\hline & $\begin{array}{l}\text { Moderate: } 0 \\
0 \%\end{array}$ & $\begin{array}{l}\text { Moderate: } 2 \\
7 \%\end{array}$ & & $\begin{array}{l}\text { Moderate: } \\
0,0 \%\end{array}$ & \\
\hline & Severe: $0,0 \%$ & $\begin{array}{l}\text { Severe: } 1 \\
3 \%\end{array}$ & & $\begin{array}{l}\text { Severe: } 0 \\
0 \%\end{array}$ & \\
\hline \multirow{5}{*}{$\begin{array}{l}\text { Aortic regurgitation } \\
\text { severity, }(n, \%)\end{array}$} & None: $22,88 \%$ & $\begin{array}{l}\text { None: } 14 \text {, } \\
47 \%\end{array}$ & None: $7,24 \%$ & $\begin{array}{l}\text { None: } 25 \\
100 \%\end{array}$ & \\
\hline & \multirow{4}{*}{$\begin{array}{l}\text { Mild: } 3,12 \% \\
\text { Moderate: } 0 \\
0 \% \\
\text { Severe: } 0,0 \%\end{array}$} & Mild: 13 & Mild: $15,50 \%$ & \multirow{3}{*}{$\begin{array}{l}\text { Mild: 0, 0\% } \\
\text { Moderate: } \\
0,0 \%\end{array}$} & \\
\hline & & \multirow{2}{*}{$\begin{array}{l}\text { Moderate: } 2 \text {, } \\
7 \%\end{array}$} & $\begin{array}{l}\text { Moderate: } 5 \text {, } \\
17 \%\end{array}$ & & \\
\hline & & & \multirow[t]{2}{*}{ Severe: $3,10 \%$} & & \\
\hline & & $\begin{array}{l}\text { Severe: } 1 \text {, } \\
3 \%\end{array}$ & & $\begin{array}{l}\text { Severe: } 0 \\
0 \%\end{array}$ & \\
\hline
\end{tabular}


$\mathrm{RL}=$ right-left leaflet non-separation, $\mathrm{RNC}=$ right-non coronary leaflet non-separation, $\mathrm{LNC}=$ left-non coronary leaflet non-separation, $\mathrm{BMI}=$ body mass index. $\mathrm{None}^{\S}=$ patients with repaired $\mathrm{CoA}$ and no residual narrowing.

Table 2

CMR measurements.

\begin{tabular}{|llllll|}
\hline & Isolated CoA & CoA+BAV & Isolated BAV & Controls & p-values \\
\hline EF\% & $65 \pm 8$ & $64 \pm 5$ & $65 \pm 8$ & $64 \pm 4$ & $p=0.91$ \\
\hline EDV $(\mathrm{ml} / \mathrm{m} 2)$ & $76 \pm 19$ & $107 \pm 48$ & $119 \pm 48$ & $75 \pm 10$ & $\mathrm{p}<0.0001$ \\
\hline ESV $(\mathrm{ml} / \mathrm{m} 2)$ & $27 \pm 8$ & $39 \pm 15$ & $42 \pm 19$ & $27 \pm 5$ & $\mathrm{p}<0.0001$ \\
\hline SV $(\mathrm{ml})$ & $51 \pm 16$ & $68 \pm 35$ & $77 \pm 32$ & $49 \pm 7$ & $\mathrm{p}<0.001$ \\
\hline LV mass $(\mathrm{g} / \mathrm{m} 2)$ & $62 \pm 26$ & $84 \pm 47$ & $106 \pm 54$ & $55 \pm 11$ & $\mathrm{p}<0.001$ \\
\hline Global Longitudinal Stain & $-17.1 \pm 2.1$ & $-17.5 \pm 2.0$ & $-16.5 \pm 2.3$ & $-18.2 \pm 2.2$ & $\mathrm{p}=0.02$ \\
\hline Global Radial Strain & $35.5 \pm 8.2$ & $35.7 \pm 6.0$ & $33.6 \pm 8.0$ & $37.0 \pm 6.2$ & $\mathrm{p}=0.16$ \\
\hline Global Circumferential Strain & $-19.9 \pm 2.7$ & $-20.1 \pm 2.2$ & $-19.5 \pm 2.4$ & $-20.4 \pm 1.9$ & $\mathrm{p}=0.49$ \\
\hline Apical Radial Strain & $47.4 \pm 14.4$ & $38.1 \pm 10.5$ & $43.9 \pm 12.2$ & $47.4 \pm 12.8$ & $\mathrm{p}=0.03$ \\
\hline Apical Circumferential Strain & $-23.2 \pm 3.7$ & $-18.3 \pm 10.7$ & $-21.3 \pm 8.2$ & $-23.8 \pm 3.0$ & $\mathrm{p}=0.02$ \\
\hline LV Sphericity Index & $0.62 \pm 0.08$ & $0.62 \pm 0.08$ & $0.59 \pm 0.06$ & $0.57 \pm 0.06$ & $\mathrm{p}=0.02$ \\
\hline LV Conicity Index & $0.52 \pm 0.07$ & $0.51 \pm 0.07$ & $0.51 \pm 0.06$ & $0.47 \pm 0.05$ & $\mathrm{p}=0.05$ \\
\hline Arch Gothicity & $1.19 \pm 0.17$ & $1.28 \pm 0.23$ & $1.32 \pm 0.30$ & - & $\mathrm{p}=0.28$ \\
\hline Arch Tortuosity & $0.46 \pm 0.07$ & $0.46 \pm 0.07$ & $0.46 \pm 0.06$ & - & $\mathrm{p}=1$ \\
\hline
\end{tabular}

$\mathrm{EF}=$ Ejection fraction, $\mathrm{EDV}=$ end-diastolic volume, $\mathrm{ESV}=$ end-systolic volume, $\mathrm{SV}=$ stroke volume.

The first five principal component analysis shape modes (Figure 4) represented $54 \%$ of the overall shape variability in the population (Table 3), and hence the corresponding shape vectors were used for statistical analyses. Different modes captured different morphological features, after careful visual assessment and correlation with morphometric measurements (Figure 5). Dominant shape features included: overall LV size (Mode 1), height (Mode 2), sphericity (Mode 3), conicity (Mode 4), and free wall movement (Mode 5). Group differences for shape modes confirmed that the isolated CoA group had the shortest and more spherical LV shape (Mode 2, $p=0.01$; Mode $3, p<0.001$ )

Mode 3 correlated inversely with all global strain measurements [i.e. $G L S(R=0.20, p=0.04)$, $G R S(R=-0.25$, $p=0.01)$ and GCS $(R=0.25, p=0.01)]$, and sphericity correlated with both global and apical radial $(R=-0.32$, $p=0.001 ; R=-0.41, p<0.0001)$ and circumferential $(R=0.32, p=0.001 ; R=0.20, p=0.04)$ strain, overall 
showing that the more spherical LVs are associated with reduced strain indices. Also, conicity inversely correlated with GRS $(R=-0.22, p=0.02)$ and $G C S(R=0.22, p=0.02)$ (Figure 6). When assessing both sphericity and conicity in a multiple regression model, only sphericity remained significantly associated with both GCS and GRS. GLS correlated with both Mode 2 and Mode 3 (Figure 7), both separately and in a multiple regression $(p=0.01)$, suggesting that there are functional implications of rounder and shorter LVs (as observed in CoA patients).

Table 3

Shape modes (M) with individual and cumulative contribution in LV variation ("Inertia \%"). This represents the \% shape variability captured by each shape mode individually and cumulatively.

\begin{tabular}{|lll|}
\hline Mode & Inertia\% & Cumulative Inertia\% \\
\hline M1 & 14.37 & 14.37 \\
\hline M2 & 13.64 & 28.01 \\
\hline M3 & 9.83 & 37.84 \\
\hline M4 & 8.37 & 46.21 \\
\hline M5 & 7.33 & 53.54 \\
\hline
\end{tabular}

When assessing the association between sphericity and strain indices in a multiple regression model accounting for the presence of AR and AS, only sphericity was significantly associated with GRS $(p=0.01)$, GCS $(p=0.01)$ and apical radial strain $(p<0.0001)$. When assessing the association between sphericity and strain indices in a multiple regression model accounting for aortic arch gothicity and tortuosity, only sphericity was associated with strain ( $p=0.05$ for GRS; we also noted $p=0.06$ for GCS).

\section{Discussion}

Left ventricular remodelling is either an adaptive response to ageing or occurs due to exposure to cardiovascular disease risk factors and myocardial injury[16]. LV sphericity index has been used in the literature as a reproducible bedside echocardiographic measure of geometric changes and a predictor of poor LV function[17, 18]. As LV sphericity index fails to detect regional apical abnormalities, a conicity index had also been introduced[11]. Statistical shape modelling is a novel tool to qualitatively and quantitatively assess geometric and functional patterns of the heart that cannot be fully captured by traditional morphometric imaging measurements. A challenge to test shape characterization in patients with myocardial infarction from the Cardiac Atlas Project has been previously carried out to provide additional information over standard clinical measures, but without in-depth interpretation of shape variations[1]. Also, LV shape in a small sample of CoA patients pre- and post-stent was compared to 
normal patients from the Cardiac Atlas Project to further describe LV remodelling through cardiac shape quantification[10].

This study focused on CMR LV shape characterization for the first time in four different groups, i.e. isolated $\mathrm{CoA}, \mathrm{CoA}+\mathrm{BAV}$, isolated BAV and healthy controls. A computational analysis was performed to compute LV average and shape deformations within the population using a methodological framework previously described by our team and colleagues $[6,15]$. Qualitative assessment of the group templates showed that patients with isolated CoA had a shorter and more spherical configuration on average, also quantitatively confirmed by a higher sphericity index. Importantly, it was found that such a configuration appeared to correlate with functional implications, as revealed by significant associations between increasing sphericity and reduced strain indices (both global and apical).

The sphericity index has been previously found to be associated with LV diastolic dimensions and able to predict systolic dysfunction better than echocardiographic measurements alone in an experimental AR model[19]. In a follow-up study of chronic AR patients to assess LV remodelling and progression, LV volumes and sphericity index were increased whereas GLS, GRS, apical rotation and twist were decreased, independent of drug therapy[18]. Of note, in our study, we observed that the relationship between sphericity and reduced strain continued to remain significant when accounting for the presence of moderate or severe AR and/or AS in a multivariable regression model. Also, in a follow-up study of clinical correlations and prognostic significance of change in LV geometric patterns, higher blood pressure, greater BMI, advancing age and male sex have been found to be key factors for developing abnormal LV geometry, whereas in our study LV geometry was independent of the above factors[20].

Another factor that could potentially be associated with the morphology and remodelling of the LV is the concurrent variant architecture of the aortic arch. Intuitively a more gothic and tortuous arch would represent higher impedance for the underlying LV, and previous observations in patients with repaired CoA suggested an association between aortic arch gothicity and tortuosity and parameters of LV function (i.e. ejection fraction, volumes and mass) $[12,21]$. However, in this population comprising patients with CoA and/or BAV, tortuosity and gothicity measurements as derived from 3D aortic shapes showed no group differences. Furthermore, multiple regression revealed that sphericity measures remained significantly associated with LV strain whilst tortuosity and gothicity did not. It should be noted that in this study all patients had a normal EF ( 65\%), whilst in previous studies exploring the functional implications of arch morphology, patients with lower EFs were also included. The functional implications of aortic arch shape remain an interesting point to explore in patients with $\mathrm{CHD}$ (including conotruncal anomalies), and previous work has suggested that, from a functional standpoint, it is not associated with a hypertensive response to exercise in patients with repaired $\mathrm{CHD}[22]$.

Strain difference correlated to shape changes might be partially explained by myocardial fiber reorientation and shortening, although this study lacks this information and future research with diffusion tensor imaging (DTI) could be very interesting in further investigating the architecture of the LV in these patients. There is a growing body of evidence showing that LV strain is not only a more sophisticated and 
thus more useful measure of LV systolic function, but that it is also more reproducible and relates to prognosis in a variety of clinical scenarios[23]. Our analysis revealed a functional implication for the observed morphological differences and therefore it may be possible to use these techniques to identify patients at higher risk of adverse remodelling, which could allow for earlier medical intervention and improved outcomes. Thus, by using this methodological framework on bigger cohort and applying clustering (i.e. patient grouping/categorization) we could then unpack the relationship between morphological and functional element and risk-stratify the groups.

Limitations: This study has the disadvantages of being a retrospective design with a relatively small number of patients per subgroup. Nevertheless, the overall population $(n=110)$ is one of the largest described using SSM, with previous studies modelling smaller populations[6, 21, 24]. Blood pressure data (cuff pressure at the time of CMR) were not available, thus a history of hypertension, as found in clinical notes, was reported instead. Finally, gothicity and tortuosity measurements were not made for the control group; a 3D sequence is a necessary requirement for this analysis but it was not part of the routine clinical imaging protocol for the healthy controls group.

\section{Conclusions}

This study illustrates computationally-derived LV templates, characterizing LV shape in both CoA and BAV patients for the first time. The analysis revealed an association between increasing LV sphericity and reduced LV strain indices, suggesting a functional implication for the observed morphological differences as well as a possible role for computational techniques to identify patients at higher risk of adverse remodelling.

\section{Abbreviations}




\begin{tabular}{ll} 
ACS & Apical circumferential strain \\
\hline ARS & Apical radial strain \\
\hline BAV & Bicuspid aortic valve \\
\hline CMR & Cardiovascular magnetic resonance \\
\hline CoA & Coarctation \\
\hline CHD & Congenital heart disease \\
\hline DTI & Diffusion tensor imaging \\
\hline EDV & End-diastolic volume \\
\hline EF & Ejection fraction \\
\hline ESV & End-systolic volume \\
\hline GCS & Global peak circumferential strain \\
\hline GLS & Global peak longitudinal strain \\
\hline GRS & Global peak radial strain \\
\hline LV & Left ventricle \\
\hline LNC & Left-non coronary \\
\hline RL & Right-left \\
\hline RNC & Right-non coronary \\
\hline SSM & Statistical shape modelling \\
\hline SV & Stroke volume \\
\hline
\end{tabular}

\section{Declarations}

Ethics approval and consent to participate: In light of the nature of the study, ethical approval was not required by the local Research \& Innovation Department.

Consent for publication: yes

Availability of data and material: Data will be made available upon request.

Competing interests: Co-author CBD is the Chief Executive Officer (part-time) of the Society for Cardiovascular Magnetic Resonance; no other competing interest to declare.

Funding: No specific funding for this study. 
Authors' contributions: Study design: FS, CBD, SC, GB; computational modelling: FS, LS; data analysis including CMR analysis: FS, GD, RL; findings interpretation: FS, CBD, NM, MH, MC, SC, GB; compiling manuscript and manuscript approval: all.

Acknowledgements: The authors gratefully acknowledge the generous support of the British Heart Foundation and the Bristol NIHR Biomedical Research Centre (BRC).

\section{References}

1. Suinesiaputra, A., et al., Statistical shape modeling of the left ventricle: myocardial infarct classification challenge. IEEE J Biomed Health Inform, 2018. 22(2): p. 503-515.

2. Ambale-Venkatesh, B., et al., Left ventricular shape predicts different types of cardiovascular events in the general population. Heart, 2017. 103(7): p. 499-507.

3. Harjai, K.J., et al., Does left ventricular shape influence clinical outcome in heart failure? Clinical Cardiology, 2000. 23(11): p. 813-819.

4. Rodrigues, J.C., et al., Comprehensive characterisation of hypertensive heart disease left ventricular phenotypes. Heart, 2016. 102(20): p. 1671-9.

5. Azhari, H., R. Beyar, and S. Sideman, On the human left ventricular shape. Comput Biomed Res, 1999. 32(3): p. 264-82.

6. Pontecorboli, G., et al., Beyond apical ballooning: computational modelling reveals morphological features of Takotsubo cardiomyopathy. Computer Methods in Biomechanics and Biomedical Engineering, 2019. 22(14): p. 1103-1106.

7. Sinning, C., et al., Bicuspid aortic valve and aortic coarctation in congenital heart disease-important aspects for treatment with focus on aortic vasculopathy. Cardiovasc Diagn Ther, 2018. 8(6): p. 780788.

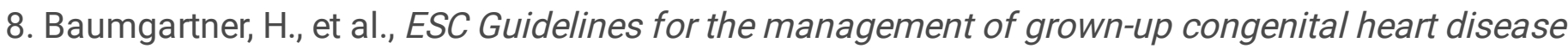
(new version 2010). Eur Heart J, 2010. 31(23): p. 2915-57.

9. Baumgartner, H., et al., 2020 ESC Guidelines for the management of adult congenital heart disease. Eur Heart J, 2021. 42(6): p. 563-645.

10. Ortiz, A., et al., Left Ventricular Shape Variation in Patients with Aortic Coarctation Pre-and Post-Stent Implantation. Journal of Investigative Medicine, 2016. 64(1): p. 201-201.

11. Di Donato, M., et al., Left ventricular geometry in normal and post-anterior myocardial infarction patients: sphericity index and 'new' conicity index comparisons. European Journal of Cardio-Thoracic Surgery, 2006. 29: p. S225-S230.

12. Sophocleous, F., et al., Aortic morphological variability in patients with bicuspid aortic valve and aortic coarctation. Eur J Cardiothorac Surg, 2018.

13. Lancellotti, P., et al., European Association of Echocardiography recommendations for the assessment of valvular regurgitation. Part 1: aortic and pulmonary regurgitation (native valve 
disease). Eur J Echocardiogr, 2010. 11(3): p. 223-44.

14. Lim, D.S. and M.A. Ralston, Echocardiographic indices of Doppler flow patterns compared with MRI or angiographic measurements to detect significant coarctation of the aorta. Echocardiography, 2002. 19(1): p. 55-60.

15. Bruse, J.L., et al., A statistical shape modelling framework to extract 3D shape biomarkers from medical imaging data: assessing arch morphology of repaired coarctation of the aorta. BMC Med Imaging, 2016. 16(1): p. 40.

16. Cohn, J.N., et al., Cardiac remodeling-concepts and clinical implications: A consensus paper from an international forum on cardiac remodeling. Journal of the American College of Cardiology, 2000. 35(3): p. 569-582.

17. Khanna, S., et al., Left Ventricular Sphericity Index is a reproducible bedside echocardiographic measure of geometric change between acute phase Takotsubo's syndrome and acute anterior myocardial infarction. ljc Heart \& Vasculature, 2020. 29.

18. Zeng, Q., et al., Left Ventricular Remodeling and Its Progression in Asymptomatic Patients with Chronic Aortic Regurgitation: Evaluation by Speckle-Tracking Echocardiography. J Am Soc Echocardiogr, 2021. 34(4): p. 360-369.

19. Roscani, M.G., et al., Left ventricular sphericity index predicts systolic dysfunction in rats with experimental aortic regurgitation. Journal of Applied Physiology, 2014. 116(10): p. 1259-1262.

20. Lieb, W., et al., The natural history of left ventricular geometry in the community: clinical correlates and prognostic significance of change in LV geometric pattern. JACC Cardiovasc Imaging, 2014. 7(9): p. 870-8.

21. Bruse, J.L., et al., How successful is successful? Aortic arch shape after successful aortic coarctation repair correlates with left ventricular function. J Thorac Cardiovasc Surg, 2017. 153(2): p. 418-427.

22. Ntsinjana, H.N., et al., Aortic arch shape is not associated with hypertensive response to exercise in patients with repaired congenital heart diseases. J Cardiovasc Magn Reson, 2013. 15: p. 101.

23. Vollema, E.M., et al., Association of Left Ventricular Global Longitudinal Strain With Asymptomatic Severe Aortic Stenosis: Natural Course and Prognostic Value. JAMA Cardiol, 2018. 3(9): p. 839-847.

24. McLeod, K., et al., Ventricular structure in ARVC: going beyond volumes as a measure of risk. Journal of Cardiovascular Magnetic Resonance, 2016. 18.

\section{Figures}

\section{Figure 1}

Statistical shape modelling (SSM) framework. Step 1: Cardiovascular magnetic resonance (CMR) cine short axis images segmentation to create 3D volume meshes of LV endocardium (in red) at end-diastole 
using Segment software; Step 2: Indication of the reconstructed shape population; Step 3: A shape mode showing a deformation as being correlated to LVEF\%, exemplifying the feasibility of statistical analysis to capture parameters of interest.

\section{Figure 2}

Morphometric measurements. a) Calculation of LV sphericity as long (A) to short (B) axis ratio, with long axis being the diameter from the mitral valve mid-point (C) to apex while short axis set as the perpendicular intersect of the long axis mid-point $(A / 2)$, and calculation of LV conicity as apical $(E)$ to short axis ratio, with apical axis being the diameter of the best fitting sphere (D) (calculations were taken directly on the scans). b) Calculation of aortic gothicity as height to width ratio (H/W), and aortic tortuosity as 1-(W/I) with I being the length of the aortic arch as shown by $\mathrm{T}$. T shows the endpoints set to indicate the aortic arch defined by using the pulmonary artery as a landmark (calculation were taken using Mimics software).

\section{Figure 3}

LV shape templates, showing the average 3D configuration of the ventricles. As labelled, the figure illustrates the average shapes of the whole population and each group separately.

\section{Figure 4}

Shape modes. Illustration of the five computed shape modes capturing LV variation (+/-2.7 SD from the mean configuration) within our population. For each mode, it is indicated what the mode overall captures, e.g. Mode 1 = size. SD = standard deviation.

\section{Figure 5}

Correlations for shape modes 3 and 4 and corresponding morphometric LV measurements (sphericity and conicity). Sphericity is significantly positively correlated with Mode 3. SD = standard deviation 
LV shape correlation with function. This figure illustrates the association between global radial and circumferential with Mode 3, sphericity and conicity.

\section{Figure 7}

Global longitudinal strain (GLS) was associated with Modes 2 and 3, showing that increased height and sphericity correlate with worse LV function. 